\title{
Search for systemic mass loss in Algols with bow shocks
}

\author{
A. Mayer ${ }^{1}$, R. Deschamps ${ }^{2,3}$, and A. Jorissen ${ }^{2}$ \\ 1 University of Vienna, Department of Astrophysics, Sternwartestraße 77, 1180 Wien, Austria \\ e-mail: a.mayer@univie.ac.at \\ 2 Institut d'Astronomie et d'Astrophysique, Université Libre de Bruxelles, CP 226, Av. F. Roosevelt 50, 1050 Brussels, Belgium \\ 3 European Southern Observatory, Alonso de Cordova 3107, 19001 Casilla, Santiago, Chile
}

Received 28 May 2015 / Accepted 24 December 2015

\begin{abstract}
Aims. Various studies indicate that interacting binary stars of Algol type evolve non-conservatively. However, direct detections of systemic mass loss in Algols have been scarce so far. We study the systemic mass loss in Algols by looking for the presence of infrared excesses originating from the thermal emission of dust grains, which is linked to the presence of a stellar wind.

Methods. In contrast to previous studies, we make use of the fact that stellar and interstellar material is piled up at the edge of the astrosphere where the stellar wind interacts with the interstellar medium. We analyse WISE W3 $12 \mu \mathrm{m}$ and WISE W4 $22 \mu \mathrm{m}$ data of Algol-type binary Be and $\mathrm{B}[\mathrm{e}]$ stars and the properties of their bow shocks. From the stand-off distance of the bow shock we are able to determine the mass-loss rate of the binary system.

Results. Although the velocities of the stars with respect to the interstellar medium are quite low, we find bow shocks present in two systems, namely $\pi$ Aqr, and $\varphi$ Per; a third system, CX Dra, shows a more irregular circumstellar environment morphology which might somehow be related to systemic mass loss. The properties of the two bow shocks point to mass-loss rates and wind velocities typical of single B stars, which do not support an enhanced systemic mass loss.
\end{abstract}

Key words. binaries: close - circumstellar matter - infrared: stars - stars: winds, outflows

\section{Introduction}

The group of Algols host stars with many different observed properties, like $\mathrm{W}$ Ser stars, $\beta$ Lyræ stars, binary $\mathrm{B}[\mathrm{e}]$ and Be stars, and symbiotic Algols, which all have in common the paradox that the donor star is more evolved but less massive than the accretor. This is achieved by mass transfer when at a certain point the mass ratio reverses. Non-conservative evolution in Algol-type binary systems has been known for 60 years (Crawford 1955). For example Chaubey (1979), Sarna (1993), and van Rensbergen et al. (2011) noted that Algol models must lose a significant fraction of their mass to reproduce observed properties. One of the most efficient scenarios that removes mass from the system is via a hotspot on the surface of the gainer ${ }^{1}$. However, no direct detection of systemic mass loss during the mass transfer process in close binaries has been reported for Algols so far.

In this work, we focus on $\mathrm{Be}$ and $\mathrm{B}[\mathrm{e}]$ stars for which binarity has been confirmed and the properties of the systems are well constrained. A Be star is a non-supergiant B star whose spectrum has, or had at some time, one or more Balmer lines in emission, and might also show infrared (IR) excess (Collins 1987). The origin of these spectral features in these binary Be stars is probably linked to the mass-transfer event (Porter \& Rivinius 2003). The IR excess is most likely caused by hot circumstellar dust (Lamers et al. 1998), which is present in the case of an evolved binary system associated with mass transfer events (e.g. Dunstall et al. 2012). B [e] stars also have strong forbidden (Fe) emission lines.

\footnotetext{
1 For an extensive explanation of the hotspot mechanism, see van Rensbergen et al. (2011) and Deschamps et al. (2013, 2015).
}

We take advantage of the fact that some of these stars are not at rest, but move with a certain speed with respect to their surrounding medium. Assuming that mass is expelled supersonically from the binary system, it is decelerated by the oncoming interstellar medium (ISM) and forms a bow shock (Baranov et al. 1971; Weaver et al. 1977). Bow shocks have been observed at all kinds of wavelengths around many stellar types, covering runaway $\mathrm{O}$ stars to asymptotic giant branch (AGB) stars (e.g. van Buren \& McCray 1988; Cox et al. 2012). In the mid-IR, these shocks are visible through thermal dust emission, when the shock front heats up dust grains at the interface between the stellar wind and the ISM (Ueta et al. 2006). A bow shock detection around an Algol is therefore direct evidence of stellar material around the binary system. In this case, the distance of the system to the apex of the bow shock can be used to derive the systemic mass-loss rate if the wind velocity, stellar velocity, and ISM density are known (Baranov et al. 1971).

This research note is a complementary study to the work done by Deschamps et al. (2015, hereafter D15), but with emphasis on the observational aspects of the systemic mass loss. Based on radiative transfer calculations, Deschamps et al. (2015, their Fig. 13) predicted the IR colour excesses expected in the case of systemic mass loss. In addition to the WISE detections of extended material around CZ Vel and SX Aur presented in D15, we discuss the properties of the circumstellar emission (CSE) of three other objects, namely CX Dra, $\pi$ Aqr, and $\varphi$ Per.

\section{WISE observations}

In D15, we performed a systematic search for extended IR emission around Algols (collected from the catalogues of Brancewicz \& Dworak 1980; and Budding et al. 2004) and Algol-related Be 
and $\mathrm{B}[\mathrm{e}]$ systems (Harmanec 2001), using archive data from the Wide-field Infrared Survey Explorer (WISE) ${ }^{2}$. WISE is an allsky survey, which mapped the sky in four bands at 3.4, 4.6, 12, and $22 \mu \mathrm{m}$ with angular resolutions of 6.' $1,6 .{ }^{\prime \prime} 4,6.5$, and $122^{\prime \prime} 0$, respectively (Wright et al. 2010). Based on the list of 70 objects (Algols and Algol-like Be stars with a WISE-source counterpart) provided by $\mathrm{D} 15^{3}$, we found that three systems, CX Dra, $\pi$ Aqr, and $\varphi$ Per, have unambiguous CSE and therefore deserve a specific analysis (in addition to CZ Vel and SX Aur, already discussed in D15).

All three objects are in the list of binary Be stars compiled by Harmanec (2001). They exhibit peculiarities that flag them as Algol candidates, or at least as systems with on-going mass transfer. With its sdO companion, $\varphi$ Per has obviously undergone a severe mass transfer, the primary and more luminous $\mathrm{B} 2[\mathrm{e}]$ component being the most massive but the least evolved. In CX Dra, mass transfer in the binary has been inferred from the variation of the polarisation with orbital phase, and from the fact that the less massive, more evolved F5III companion fills its Roche lobe (Berdyugin \& Piirola 2002). In $\pi$ Aqr photometric, spectroscopic (broad and complex $\mathrm{H} \alpha$ line profile), and polarimetric variations observed during the second half of the 20th century are tentatively attributed to variable mass transfer between the binary components (Bjorkman et al. 2002; Hanuschik et al. 1996).

The extended emission around the stars was detected in Band 3 (W3) at $12 \mu \mathrm{m}$ (CX Dra, $\pi$ Aqr) and Band 4 (W4) at $22 \mu \mathrm{m}$ (CX Dra, $\pi$ Aqr, $\varphi$ Per). For the two objects with CSE detected in both bands, WISE W4 offers greater details, most likely because the thermal emission of the shock-heated dust grains peaks at longer wavelengths (Draine 1981). In the following, the circumstellar material (CSM) morphology of the three objects is described. Figures 1-5 depict the WISE images of CX Dra, $\pi$ Aqr, and $\varphi$ Per, while Table 1 provides their stellar properties.

\subsection{Dra}

CX Dra (HIP 92133) is a 6.696 d period Algol B2.5Ve+F5III system at a distance of $396 \mathrm{pc}$ (van Leeuwen 2007); one of the components rotates rapidly. Although it is not eclipsing, Berdyugin \& Piirola (2002) estimate the mass of the two components to be $3.9 M_{\odot}$ and $0.9 M_{\odot}$ at $i=70^{\circ}$. The authors, however, correctly note that these masses are too small to match the spectral types of the two stars.

The circumstellar environment of the star is shown in Figs. 1 and 2. The emission in the WISE W4 $22 \mu \mathrm{m}$ band is concentrated to the east of the star and is traceable to a distance of about $120^{\prime \prime}$ (47500 au at $396 \mathrm{pc}$ ). The morphology of the CSM is somewhat puzzling because several aspects are not in favour of an ISM interaction. First, the direction of the proper motion is S-E, but the shape of the emission is not symmetric and is more concentrated $\mathrm{N}-\mathrm{E}$ of the star. Second, the emission is not detached from the star and the flux seems to decrease with distance, which is not expected for a bow shock where the brightest region is at the position of the shock front.

Furthermore, the CSM of CX Dra on the WISE image describes an arc emerging east of the star and curved towards the north. Similar arcs are found to be part of an Archimedean spiral which is caused by a semi-detached companion interacting with

\footnotetext{
2 The IRSA:WISE archive can be found at http://irsa.ipac. caltech.edu/applications/wise/

3 The list of objects can be found in Appendix A.
}
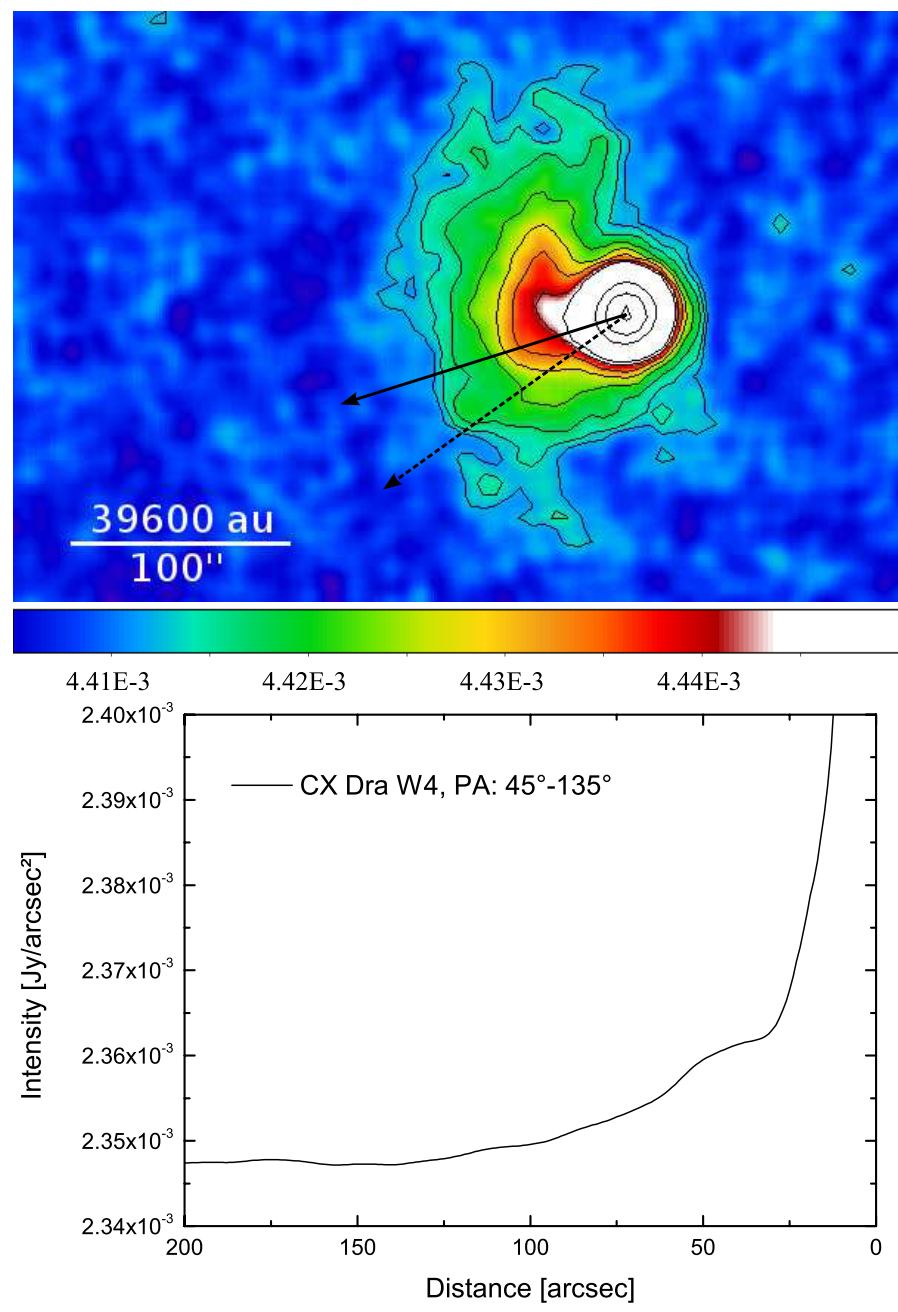

Fig. 1. Upper panel: WISE W4 image of CX Dra at $22 \mu \mathrm{m}$. The continuous black arrow gives the uncorrected proper motion from the reprocessed HIPPARCos catalogue (van Leeuwen 2007), while the dashed arrow points to the direction of the space motion corrected from the solar motion (Coşkunoğlu et al. 2011). The values of the motion are given in Table 1. The values of the colour bar are given in $\mathrm{Jy} \mathrm{pix}^{-1}$. Lower panel: Integrated intensity cut through a wedge covering position angles (PA): $45^{\circ}-135^{\circ}$.

the wind of a primary (e.g. Mastrodemos \& Morris 1999; Mayer et al. 2011; Maercker et al. 2012). The spacing of the spiral arms is thereby defined by the wind velocity of the mass losing star and the orbital period of the companion. However, assuming a wind velocity of $1000 \mathrm{~km} \mathrm{~s}^{-1}$ for the B2.5Ve star and an orbital period of $6.696 \mathrm{~d}$ for the F5III companion, the resulting spiral spacing is $3.87 \mathrm{au}$, which is several orders of magnitude smaller than what is seen on the WISE image. For comparison, the pixel size of the image is $1^{\prime \prime} 375$, which is 545 au at $396 \mathrm{pc}$. This implies that a spiral formed by the B2.5Ve+F5III system would show 140 windings per WISE W4 pixel. The observed arc is therefore not related to this shaping mechanism.

In the colour-colour diagram shown in Fig. 13 of D15 that depicts the WISE W4/W1 against 2MASS $J / K_{\mathrm{S}}$ flux ratios, CX Dra is located only slightly above the black-body curve $\left(F_{\mathrm{J}} / F_{\mathrm{K}_{\mathrm{s}}}=1.678, F_{\mathrm{W} 4} / F_{\mathrm{W} 1}=0.047\right)$. Many other objects fall into this region of the diagram and no peculiarity can be drawn from it. Still, there is no doubt that extended emission is present in the WISE W4 image.

In the shorter WISE W3 band at $12 \mu \mathrm{m}$ (see Fig. 2), CX Dra also shows extended emission east of the star but in much less 

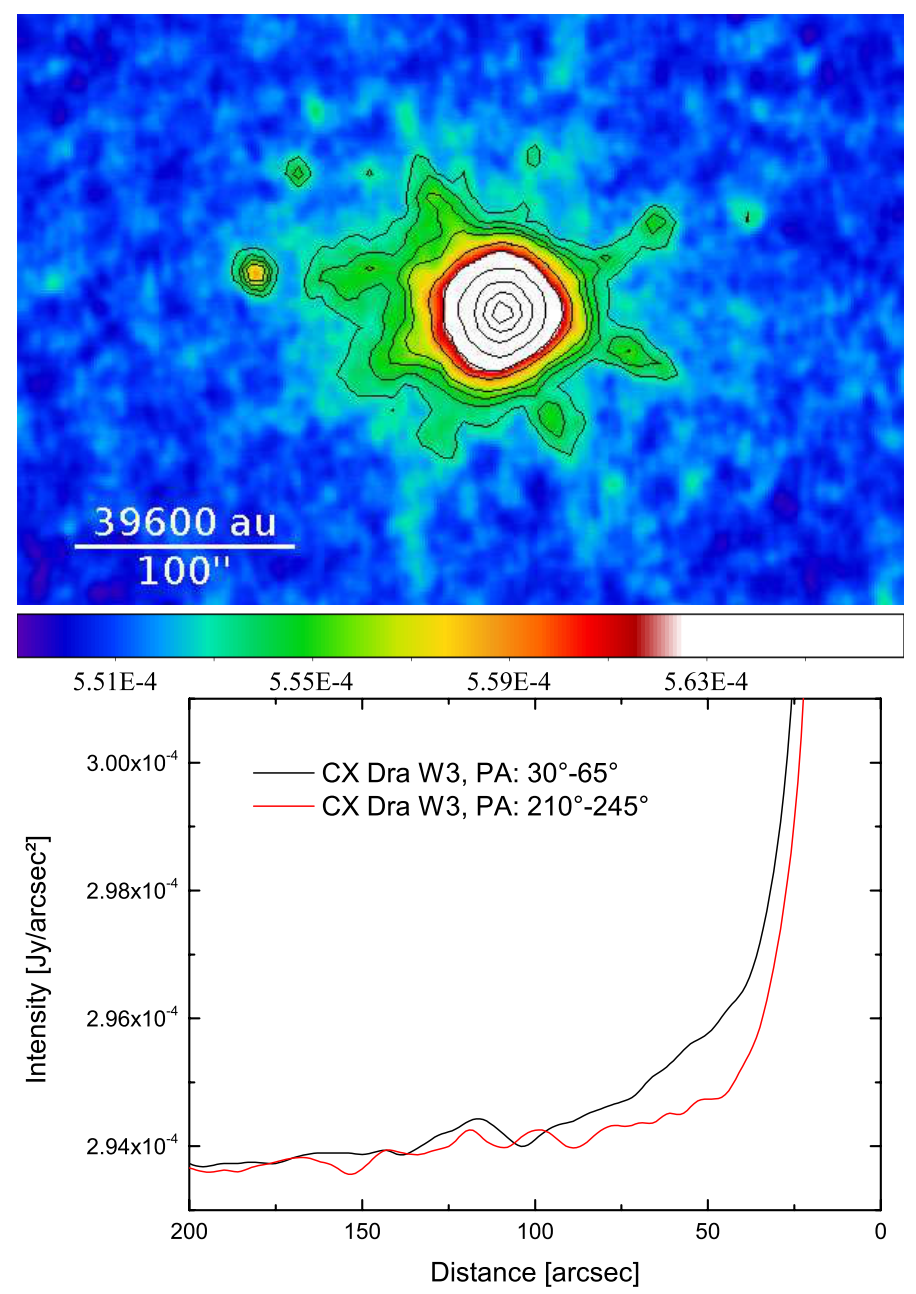

Fig. 2. Upper panel: WISE W3 images of CX Dra at $12 \mu \mathrm{m}$. Lower panel: integrated intensity cuts through a wedge covering PA: $30^{\circ}-65^{\circ}$ (black line) where emission is visible and PA: $210^{\circ}-245^{\circ}$ (red line) without extended emission. We had to choose smaller wedges to avoid the flux being dominated by the diffraction spikes of the PSF.

detail than in the W4 image. The detection of emission in W3 and W4, however, allows us to estimate the temperature of the dust emission around CX Dra. We performed aperture photometry on a circle of radius $15^{\prime \prime}$ in both bands. The region we chose is centred at a distance of $56^{\prime \prime}$ from the star at PA $=48^{\circ}$ and falls between the diffraction spikes of the PSF which is dominating the $\mathrm{W} 3$ image. The resulting fluxes are $F_{v, 12}=0.207 \mathrm{Jy}$ and $F_{v, 22}=1.650 \mathrm{Jy}$ at $12 \mu \mathrm{m}$ and $22 \mu \mathrm{m}$, respectively. Adopting the corresponding absorption coefficients of astronomical silicates $Q_{\mathrm{abs}, 12}=5.60 \times 10^{-2}$ and $Q_{\mathrm{abs}, 22}=3.39 \times 10^{-2}$ (Draine $1985)$, the $12 \mu \mathrm{m}$ and $22 \mu \mathrm{m}$ fluxes correspond to a temperature of $124 \mathrm{~K}$ (for details see Jorissen et al. 2011).

Since no other archival observations are available for CX Dra, we cannot conclude on the shaping mechanism of its CSM. We note, however, that the star might be a possible candidate for showing systemic mass loss in its circumstellar environment, but further observations are needed.

\section{2. $\pi$ Aqr}

Pi Aquarii (HIP 110672) is a 84.1 d period binary located $240 \mathrm{pc}$ from the sun (van Leeuwen 2007). The system comprises a rapidly rotating $\mathrm{B} 1 \mathrm{Ve}$ star at the origin of the Be phenomenon and an A-F type companion. Bjorkman et al. (2002) estimate
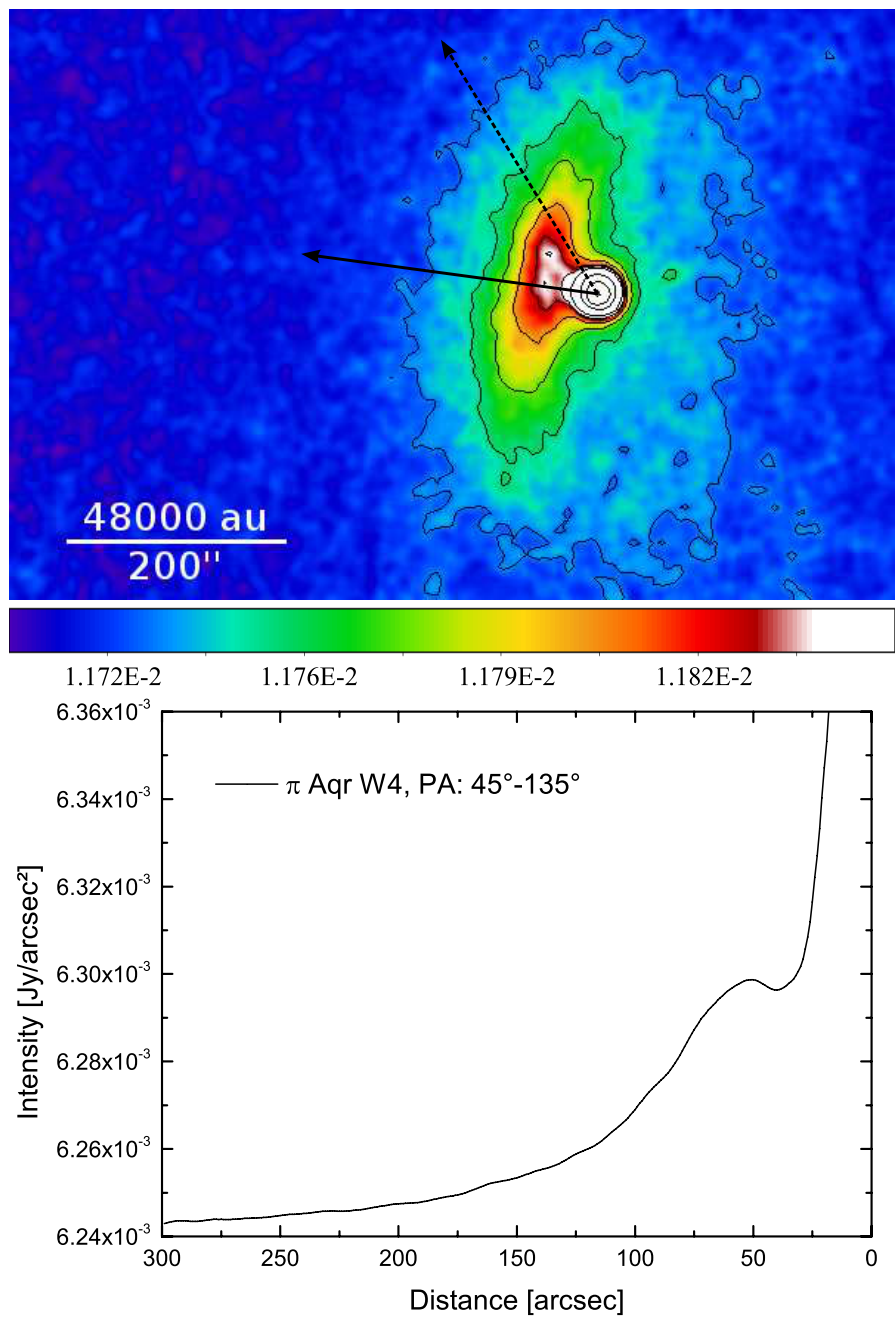

Fig. 3. Same as Fig. 1 for $\pi$ Aqr. The integrated intensity cut in the lower panel covers PA: $45^{\circ}-135^{\circ}$.

the mass of the components to be $M_{1} \sin ^{3} i=12.4 M_{\odot}$ and $M_{2} \sin ^{3} i=2.0 M_{\odot}$ with an orbital inclination $i=(50-75)^{\circ}$. The stellar wind is one of the fastest among the Be stars with a terminal velocity of $1450 \mathrm{~km} \mathrm{~s}^{-1}$; the mass-loss rate is one of the highest with $\dot{M}=2.61 \times 10^{-9} M_{\odot} \mathrm{yr}^{-1}$ estimated from the Si IV profile (Snow 1981). We note that the Si IV lines likely form inside the Roche lobe of the gainer star and might therefore not trace the systemic mass-loss rate.

The WISE W4 image of $\pi$ Aqr is depicted in Fig. 3. The emission shows a morphology that is typical for a wind-ISM interaction with a bow shock in the direction of the space motion. The bow shock cone is quite symmetric on the northern and southern half, extending to about $220^{\prime \prime}$ (52 800 au at $240 \mathrm{pc}$ ) in those directions. In the direction of motion, the material can be traced to about 150" (36000 au) from the binary system. The emission peak, however, is closer to the system at about $52^{\prime \prime}$ (12480 au).

In WISE W3 at $12 \mu \mathrm{m}$ (see Fig. 4), the CSE is concentrated to the east of the star at the same position where the bow shock in W4 is visible, but not as extended in the north-south direction. The lower panel of Fig. 4 shows cuts through regions with and without extended emission.

In the same manner as for CX Dra, we also performed aperture photometry $\left(r=15^{\prime \prime}\right)$ for $\pi$ Aqr in both bands at a region centred at a distance of $61^{\prime \prime}$ from the star at PA $=46^{\circ}$. The 

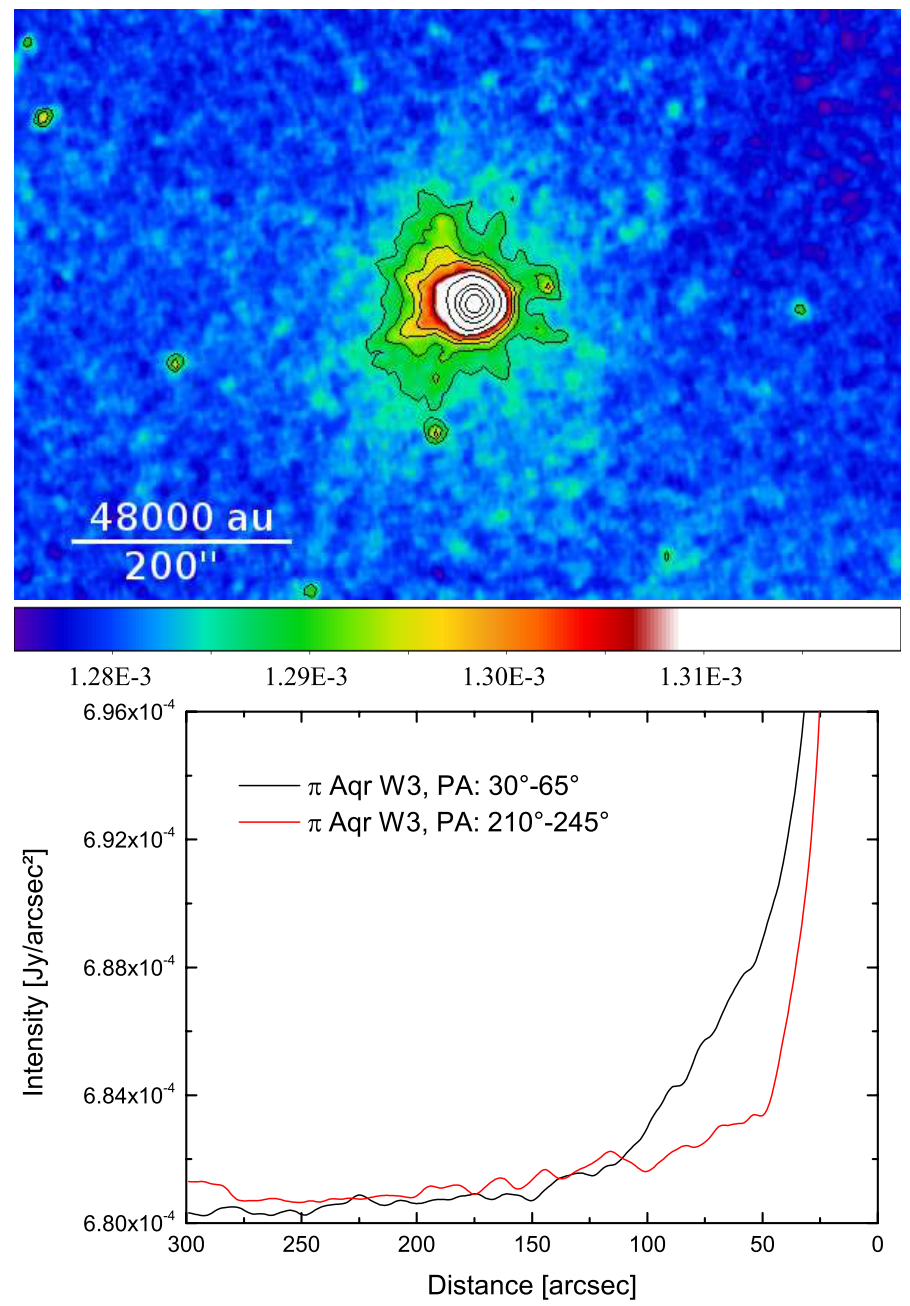

Fig. 4. Same as Fig. 2 for $\pi$ Aqr at $12 \mu \mathrm{m}$. The integrated intensity cuts in the lower panel cover PA: $30^{\circ}-65^{\circ}$ (black line) where emission is visible and PA: $210^{\circ}-245^{\circ}$ (red line) without extended emission.

resulting fluxes are $F_{v, 12}=0.481 \mathrm{Jy}$ and $F_{v, 22}=4.411 \mathrm{Jy}$ at $12 \mu \mathrm{m}$ and $22 \mu \mathrm{m}$, respectively. Adopting the same absorption coefficients of astronomical silicates as for CX Dra, the $12 \mu \mathrm{m}$ and $22 \mu \mathrm{m}$ fluxes correspond to a temperature of $120 \mathrm{~K}$.

\section{3. $\varphi$ Per}

Phi Persei (HIP 8068) is a long period Algol B2[e]+sdO system $\left(P_{\text {orb }}=127 \mathrm{~d}\right)$ at a distance of $220 \mathrm{pc}$ (van Leeuwen 2007). The system is likely at the end of its mass-transfer phase (Gies et al. 1998) and the material transferred from the donor star has largely spun up the gainer star (primary) to the rotation rate now observed. Based on double-line spectroscopic orbital elements, the masses of the components have been estimated to be $9.3 \pm 0.3 M_{\odot}$ for the $\mathrm{B}[\mathrm{e}]$ primary and $1.14 \pm 0.04 M_{\odot}$ for the sdO secondary (donor star). A hotspot region detected on the edge of the disc produces strong Fe IV lines. The envelope of the companion has mostly been stripped off by the Roche-lobe overflow (RLOF) event and the secondary, now a hot sdO star, is only visible in the UV.

The WISE W4 $22 \mu \mathrm{m}$ emission of $\varphi$ Per is shown in Fig. 5. The CSM is elliptically shaped with the major axis approximately in the N-S direction. The extent of the emission to the south is about $290^{\prime \prime}(63800 \mathrm{au}$ at $220 \mathrm{pc})$, while the frame is cut off in the north $250^{\prime \prime}$ from the star. East of the star at $\approx 100^{\prime \prime}$
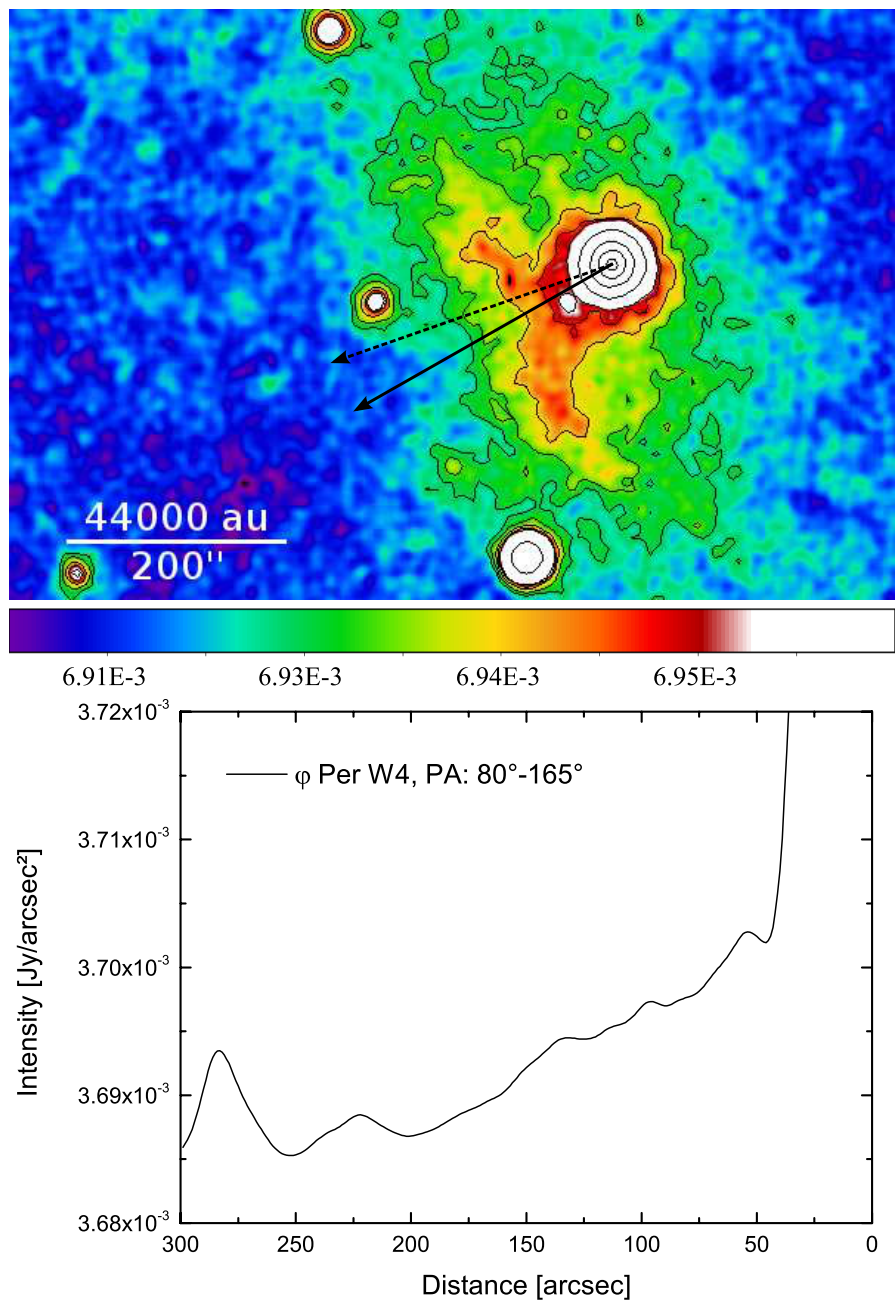

Fig. 5. Same as Fig. 1 for $\varphi$ Per. The integrated intensity cut in the lower panel covers PA: $80^{\circ}-165^{\circ}$. The peaks at distances of about $223^{\prime \prime}$ and $283^{\prime \prime}$ in the lower panel correspond to the stars visible in the image at position angles of $99^{\circ}$ and $164^{\circ}$, respectively.

(22 $000 \mathrm{au}$ ), a brightened bar is visible with the same N-S orientation as the whole elliptical emission and a length of about $240^{\prime \prime}$ $(52800 \mathrm{au})$. The bar is bent towards the star at the same position angle as the direction of the space motion, which indicates that this is the interface where the ISM interacts with the stellar material. These bendings are visible in hydrodynamic simulations of bow shocks where the shocked stellar and ambient material cool efficiently (see Fig. 15 in Comeron \& Kaper 1998). A beautiful example of a bent bow shock is found around the AGB star X Her (Jorissen et al. 2011). In contrast to the other two objects, $\varphi$ Per does not show extended emission in WISE W3.

\section{Bow shock properties and systemic mass loss}

For a star that moves with respect to its surrounding medium, the stellar motion adds an asymmetry to the wind velocity profile, since different parts of the wind face the ISM with different relative velocities. If the motion is supersonic, a bow shock arises at the interface where the ram pressure of the ISM and the stellar wind balance. The stand-off distance, i.e. the distance of the star to the apex of the shock front, is given by

$R_{0}=\sqrt{\frac{\dot{M} v_{w}}{4 \pi \rho_{0} v_{*}^{2}}}$, 
Table 1. Stellar properties.

\begin{tabular}{l|rrr}
\hline \hline & CX Dra & $\pi$ Aqr & $\varphi$ Per \\
\hline Spec. type & B2.5Ve+F5III & B1 Ve+[A-F] & B2[e]+sdO \\
$M_{1}\left[M_{\odot}\right]$ & 3.9 & $14.0 \pm 1.0$ & $9.3 \pm 0.3$ \\
$M_{2}\left[M_{\odot}\right]$ & 0.9 & 2.3 & $1.1 \pm 0.1$ \\
$P_{\text {orb }}[\mathrm{d}]$ & 6.696 & 84.1 & 127 \\
$\mathrm{D}[\mathrm{pc}]$ & $396 \pm 35$ & $240 \pm 15$ & $220 \pm 9$ \\
$\mathrm{z}[\mathrm{pc}]$ & 149 & 169 & 43 \\
$n_{\mathrm{H}}\left[\mathrm{cm}^{-3}\right]$ & 0.45 & 0.37 & 1.30 \\
$\mathrm{RV}\left[\mathrm{km} \mathrm{s}^{-1}\right]$ & $-2.1 \pm 2.3$ & $-4.9 \pm 0.1$ & $-4.0 \pm 2.1$ \\
\hline$v_{*}\left[\mathrm{~km} \mathrm{~s}^{-1}\right]$ & $21.1 \pm 2.0$ & $21.0 \pm 1.1$ & $29.8 \pm 1.6$ \\
$\mathrm{PA}\left[{ }^{\circ}\right]$ & $107.6 \pm 1.2$ & $82.3 \pm 0.6$ & $119.7 \pm 0.2$ \\
$i\left[{ }^{\circ}\right]$ & $-5.7 \pm 63.1$ & $-13.5 \pm 1.2$ & $-7.7 \pm 30.3$ \\
\hline$v_{* \text { LSR }}\left[\mathrm{km} \mathrm{s}^{-1}\right]$ & $25.5 \pm 2.1$ & $13.5 \pm 1.0$ & $13.1 \pm 1.7$ \\
$\mathrm{PA}_{\mathrm{LSR}}\left[{ }^{\circ}\right]$ & $125.7 \pm 1.0$ & $31.5 \pm 0.5$ & $109.4 \pm 0.6$ \\
$i_{\mathrm{LSR}}\left[{ }^{\circ}\right]$ & $32.4 \pm 11.4$ & $6.6 \pm 3.7$ & $-4.4 \pm 90$ \\
\hline Ref. & 1 & $2,3,4$ & 5 \\
\hline
\end{tabular}

Notes. The space velocities were calculated following Johnson \& Soderblom (1987) using the HIPPARCos proper motion and parallax (van Leeuwen 2007) and radial velocities from the catalogue by de Bruijne \& Eilers (2012). The solar motion adopted to convert heliocentric motion into LSR motion is $(U, V, W)=(8.50 \pm 0.29$, $13.38 \pm 0.43,6.49 \pm 0.26) \mathrm{km} \mathrm{s}^{-1}$ (Coşkunoğlu et al. 2011).

References. (1) Berdyugin \& Piirola (2002); (2) Zharikov et al. (2013); (3) Linnell et al. (1988); (4) Bjorkman et al. (2002); (5) Hummel \& Štefl (2001).

where $v_{w}$ is the terminal wind velocity, $v_{*}$ the stellar velocity with respect to the ISM, $\dot{M}$ the mass-loss rate, and $\rho_{0}$ the density of the surrounding medium (Baranov et al. 1971). The density can be expressed in number density of hydrogen atoms $\left(m_{\mathrm{H}}=\right.$ $1.6727 \times 10^{-27} \mathrm{~kg}$ ), which follows roughly

$n_{\mathrm{H}}=2.0 \mathrm{e}^{-\frac{|z|}{100 \mathrm{pc}}}$

where $z$ is the galactic height (Mihalas \& Binney 1981) and $n_{\mathrm{H}}$ is given in atoms per $\mathrm{cm}^{3}$. Wilkin (1996) demonstrated that the shape of the bow shock only depends on the stand-off distance, while Cox et al. (2012) showed that this assumption remains valid for viewing angles up to $70^{\circ}$. Above this value, the bow shock cone becomes broader. Therefore, we were able to use Eq. (1) to estimate the mass-loss rate from the binary system by measuring the stand-off distance. Generally, the ISM density and stellar velocity can be determined following Eq. (2) and Johnson $\&$ Soderblom (1987), respectively. While the error of the space motion is negligible, the ISM density value is only an estimate since the star could move through a dense cloud, which is not considered by Eq. (2). The respective values of these quantities for the three objects are given in Table 1. To obtain the space motion $\left(v_{*, \mathrm{LSR}}\right)$ with respect to the local standard of rest, we corrected the heliocentric motions from the solar motion vector $(U, V, W)_{\odot}=(8.50 \pm 0.29,13.38 \pm 0.43,6.49 \pm 0.26) \mathrm{km} \mathrm{s}^{-1}$ (Coşkunoğlu et al. 2011). However, since the proper motions are quite small (a few mas $\mathrm{yr}^{-1}$ ), the correction for the solar motion has a large impact, especially on the PA of the motion. Interestingly, the PA of the corrected LSR motion is a worse match to the bow-shock orientation than the PA of the uncorrected motion (see Figs. 1, 3, and 5). Using $(U, V, W)_{\odot}=$ $(10.00 \pm 0.36,5.25 \pm 0.62,7.17 \pm 0.38) \mathrm{km} \mathrm{s}^{-1}$ determined from the HIPPARCos data by Dehnen \& Binney (1998) leads to $v_{\text {LSR }}$ velocities which are slightly closer to the better matching heliocentric values. A similar discrepancy between bow-shock orientation and LSR motion (and less so with heliocentric motion) is found by Peri et al. $(2012,2015)$ for a large number of $\mathrm{O}$ - and B-type stars with bow shocks, as collected in the WISE E-BOSS survey. For this reason, we overplotted both the heliocentric and LSR motions on the WISE images in Figs. 1, 3, and 5.

Sarna (1993), van Rensbergen et al. (2011), and more recently Deschamps et al. $(2013,2015)$ have suggested that Algols lose a significant fraction of their initial mass during the masstransfer phase. van Rensbergen et al. (2011) state that a hotspot mechanism may be invoked to remove up to $15 \%$ of the binarysystem initial mass. In this scenario, those parts of the stellar surface hit by the RLOF stream (the hotspot) emit radiation whose pressure triggers mass loss at a rate of up to $10^{-5} M_{\odot} \mathrm{yr}^{-1}$. Such a high mass-loss rate would change the size of the bow shock considerably. Moreover, a stream winding around the binary system forms (see Fig. 1 of D15), possibly altering the shape of the bow shock as well.

In order to identify the origin of the mass causing the observed bow shocks, one has to evaluate the dynamical age of the bow shock. The distance of the bow shocks to the central system is 22000 au and 36000 au for $\varphi$ Per and $\pi$ Aqr, respectively. If the bow shocks are caused by systemic mass loss triggered by a hot spot, the wind velocity is about $1000 \mathrm{~km} \mathrm{~s}^{-1}$ (see Fig. 3 of D15) and the resulting kinematic ages of the bow shocks are of the order of $100 \mathrm{yr}$, much shorter than the duration of the masstransfer phase in Algol systems (10 $10^{5}$ r; Deschamps et al. 2013). Therefore, for Algols currently in the rapid mass-transfer phase, if a bow shock is present it will stay for the whole duration of the mass transfer.

Since the wind velocities of $\pi$ Aqr and $\varphi$ Per are not known, we cannot directly use Eq. (1) to relate the bow-shock stand-off distance to the mass-loss rate causing the bow shock and then compare the latter with the predictions of systemic mass-loss rates from the hotspot scenario of D15. Nevertheless, the relation between the mass-loss rate and the wind velocity may help us to evaluate the likelihood of systemic mass loss triggered by the hotspot scenario. In these calculations, we used the ISM densities $\rho_{0}=n_{\mathrm{H}} \times m_{\mathrm{H}}$ and space velocities $v_{* \text {,LSR }}$ listed in Table 1 with stand-off distances of $R_{0}=36000$ au for $\pi$ Aqr and $R_{0}=22000$ au for $\varphi$ Per, as measured on the WISE images. Figure 6 depicts the relationship between mass-loss rate and wind velocity for $\pi$ Aqr. The colours show the differences in the calculated $R_{0}$ to the observed value of $36000 \mathrm{au}$.

For an expected wind velocity between 700 and $1500 \mathrm{~km} \mathrm{~s}^{-1}$, only a small range of mass-loss rates from $4 \times 10^{-10} M_{\odot} \mathrm{yr}^{-1}$ to $4 \times 10^{-9} M_{\odot} \mathrm{yr}^{-1}$ can match the observed stand-off distance $( \pm 5000 \mathrm{au})$ of the bow shock around $\pi$ Aqr. This range of massloss rates is well below the systemic mass-loss rate inferred from the hotspot scenario (of the order of $10^{-5} M_{\odot} \mathrm{yr}^{-1}$ ). Such a high mass-loss rate in combination with a wind velocity of $\approx 1000 \mathrm{~km} \mathrm{~s}^{-1}$ would cause a stand-off distance that is a factor of 100 larger than observed. To bring the wind velocity in line with the proposed $\dot{M}$ from the hotspot scenario and with the observed $R_{0}$, it has to decrease almost to 0 . In other words, it is highly unlikely that a systemic mass loss of the order of $10^{-5} M_{\odot} \mathrm{yr}^{-1}$ is currently present in $\pi$ Aqr. If systemic mass loss is ongoing, it does not exceed $10^{-8} M_{\odot} \mathrm{yr}^{-1}$. We emphasise, however, that the mass-loss rate of $\pi$ Aqr inferred from the bow-shock properties fits well the value observed for the Be star $\left(2.61 \times 10^{-9} M_{\odot} \mathrm{yr}^{-1}\right.$; Snow 1981). We obtain similar results For $\varphi$ Per: $\dot{M}=2 \times 10^{-10}-6 \times 10^{-9} M_{\odot} \mathrm{yr}^{-1}$ for $v_{\mathrm{w}}=700$ $1500 \mathrm{~km} \mathrm{~s}^{-1}$ for a measured $R_{0}$ of $22000 \pm 5000 \mathrm{au}$.

The Be star wind as the origin of the bow shock is further demonstrated by the fact that the bow shocks discovered in the present study are restricted to early-type B stars (Figs. 7, 8). Our 


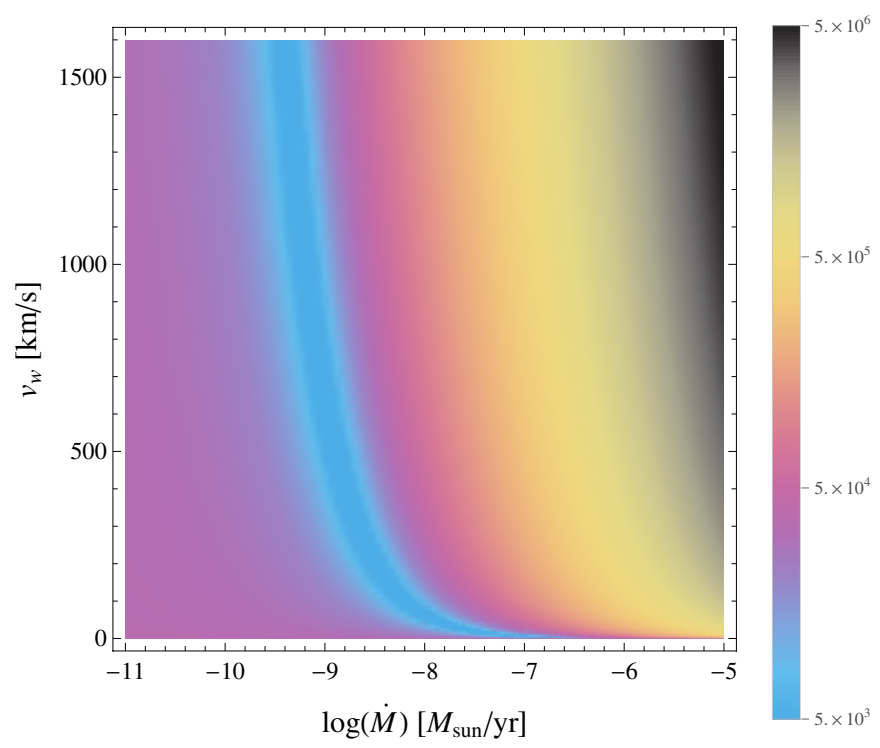

Fig. 6. Density plot relating the mass-loss rate and wind velocity of $\pi$ Aqr to the bow-shock stand-off distance $R_{0}$. The colours indicate the difference in au to the measured $R_{0}=36000$ au.

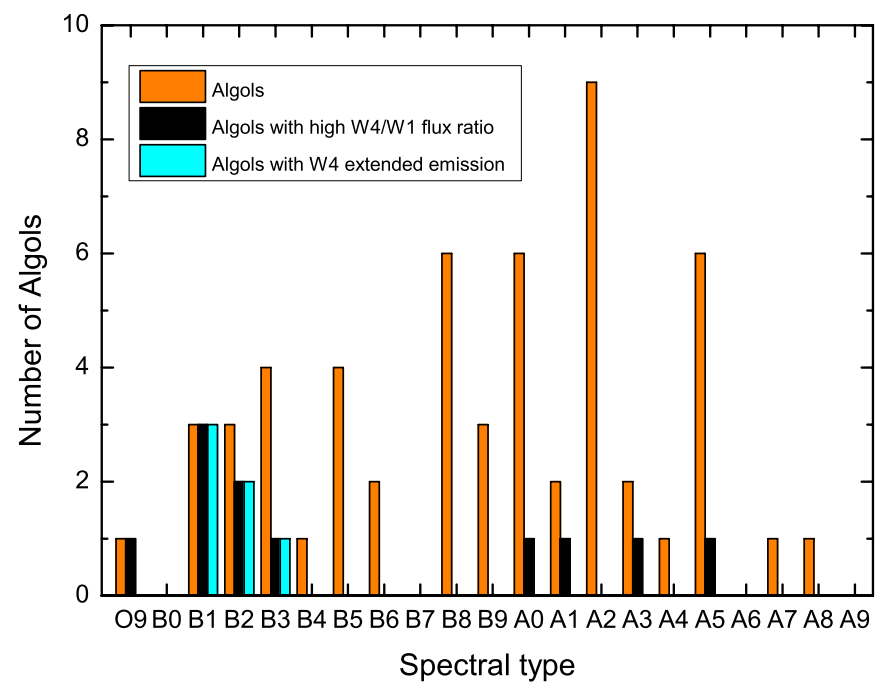

Fig. 7. Distribution of Algols among early spectral types (orange bars), along with those with detected extended emission in the WISE W4 band (cyan bars) and with W4/W1 flux ratios falling above the black-body line (black bars).

sample also includes a O9.7Ibe star, RY Sct, which shows a high WISE W4/W1 flux ratio. However, RY Sct is much farther away than the three B stars discussed in the present paper since it has a parallax not significantly different from zero (van Leeuwen 2007); hence, if present, the extended emission would be hardly detectable. Given that the $V$ magnitude of RY Sct (O9.7Ibe) amounts to 9.1, compared to $V=4.6$ for $\pi \operatorname{Aqr}(\mathrm{B} 1 \mathrm{Ve})$, RY Sct is located at least a factor of 10 farther away than $\pi$ Aqr. All other parameters being equal, the bow shock of RY Sct would thus only extend up to $15^{\prime \prime}$ from the star, and would not be resolved by WISE. This conclusion is supported by Fig. 8, which reveals that the Be stars with a detected bow shock are the nearest and the warmest in the sample.

The second Be star with extended emission that we did not include in this study is the B2Vne star V696 Mon. The WISE W4 image is depicted in the upper panel of Fig. 9 and shows

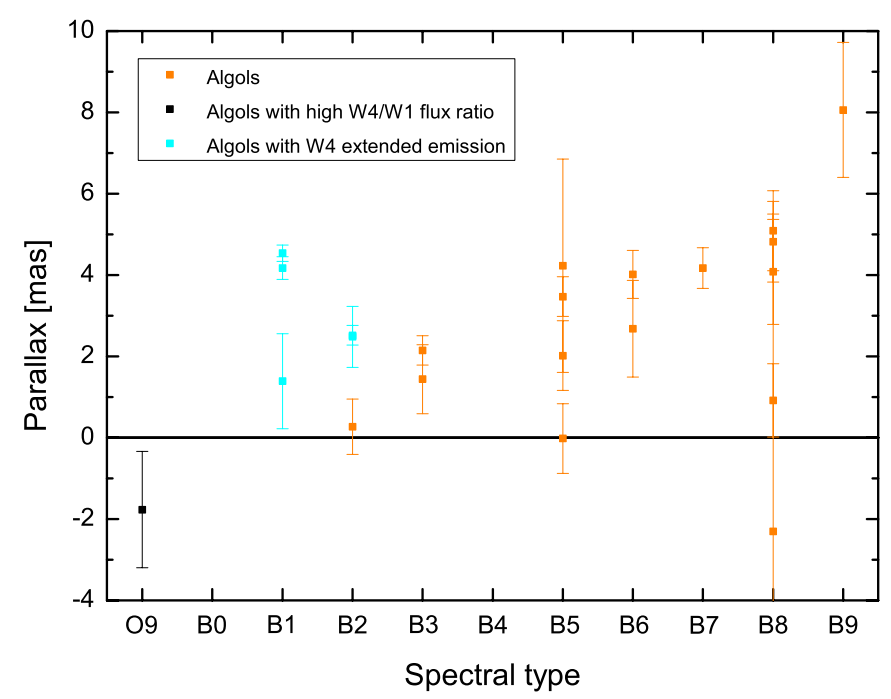

Fig. 8. Distance distribution of Algols earlier than spectral type A. Orange squares are Algols without detection of CSM, cyan squares show those with detected extended emission in the WISE W4 band, and black squares those with W4/W1 flux ratios falling above the blackbody line (according to Fig. 13 of D15).

a peculiar morphology around the star. The extended emission reaches north of V696 Mon and seems to engulf the star BD $06^{\circ} 1393$ located $148^{\prime \prime}$ from V696 Mon at PA $=8^{\circ}$. This association is probably not real, since the Tycho- 1 parallaxes of the two stars are quite different. Although the space velocity of V696 Mon $\left[v_{* \text { LSR }}=(15.8 \pm 6.2) \mathrm{km} \mathrm{s}^{-1}\right]$ is comparable to the three stars studied here and the IR emission seems to be aligned with the direction of the space motion, the upstream structure does not resemble a bow shock.

We also note that the surrounding ISM of V696 Mon is extremely rich (the star-forming region Monoceros R2 is 56' away) as seen in the IRAS $100 \mu \mathrm{m}$ image (lower panel of Fig. 9), and it is difficult to conclude whether the WISE $22 \mu \mathrm{m}$ emission originates from CSM or ISM.

\section{Conclusion}

We studied a sample of 70 Algol and Algol-like Be systems with entries in the WISE catalogue ${ }^{4}$ with the aim of identifying those Algol systems surrounded by dust left over by systemic mass loss. In D15, the two objects, CZ Vel and SX Aur, were discussed, and here we find that three new objects, CX Dra, $\pi$ Aqr, and $\varphi$ Per, show CSM in the WISE W4 band at $22 \mu \mathrm{m} ; \pi$ Aqr and CX Dra also show material in WISE W3 at $12 \mu \mathrm{m}$. The two objects $\pi$ Aqr and $\varphi$ Per show clear evidence of an interaction of CSM with the ISM. For these events, we used the distance of the star to the bow shock to derive the mass-loss rate of the matter that escapes from the system. For both systems we estimate a mass-loss rate of $\approx 10^{-9} M_{\odot} \mathrm{yr}^{-1}$, which is comparable to the mass-loss rate found for single B-type stars and much lower than the predicted systemic mass-loss rate from the hotspot scenario $\left(\sim 10^{-5} M_{\odot} \mathrm{yr}^{-1}\right.$, van Rensbergen et al. 2011; Deschamps et al. 2013, 2015). Incidentally, several bow shocks have been reported around single Be stars by e.g. Kobulnicky et al. (2012), Peri et al. (2012, 2015), and Noriega-Crespo et al. (1997). It

\footnotetext{
4 We checked the Herschel Science Archive as well, but only 2 of the 70 targets had been observed by the Herschel Space Observatory, neither of which showed CSE.
} 

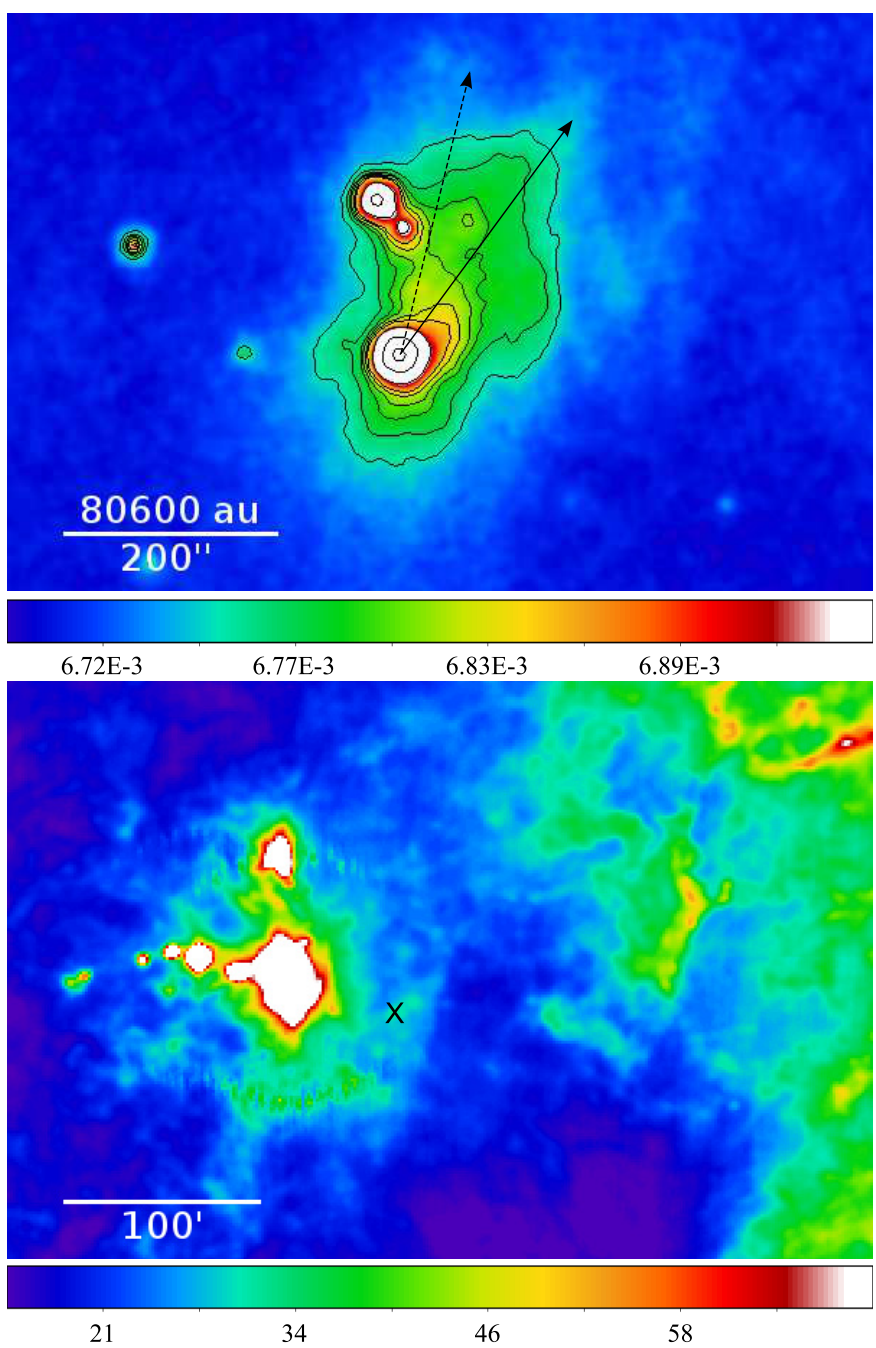

Fig. 9. Upper panel: WISE W4 image of V696 Mon at $22 \mu \mathrm{m}$. The continuous black arrow gives the uncorrected proper motion from the reprocessed HIPPARCOS catalogue (van Leeuwen 2007), while the dashed arrow points to the direction of the space motion corrected from the solar motion (Coşkunoğlu et al. 2011). The colour bar values are given in $\mathrm{Jy} \mathrm{pix}^{-1}$. Lower panel: IRAS $100 \mu \mathrm{m}$ image of the surroundings of V696 Mon (marked as an X). The colour bar values are given in $\mathrm{MJy} \mathrm{sr}^{-1}$.

seems most likely, therefore, that the bow shocks reported here are unrelated to the binary nature of the considered Be stars, and are of the same origin as those observed among non-binary Be stars. However, this does not entirely exclude the existence of systemic mass loss in these systems. It could well be that the mass transfer episode has already come to an end and the systemic mass-loss rate has dropped.

Therefore, the prospects of finding observational support for systemic mass loss in Algols seem dark. Of the initial sample of 70 targets, only a handful of stars appear to exhibit IR excesses (see Figs. 1-5 and Figs. 13, 14 of D15). It is conceivable that the simulations of D15 overestimate the amount of dust that survives the hotspot mechanism. For the two stars that show the presence of CSM expressed by a bow shock ( $\pi$ Aqr and $\varphi$ Per), neither shock appears to be tied to systemic mass loss.

In the case of CX Dra, we were not able to identify the shaping mechanism responsible for the asymmetric CSE. Both common triggers for asymmetries, ISM interaction forming a bow shock and binary interaction forming an Archimedean spiral, can be excluded for various reasons. We note therefore that this system is an interesting case for further observations since it may be a case where mass lost from the system is visible in the circumstellar environment.

Acknowledgements. We want to thank the anonymous referee for the constructive and helpful remarks. This research is supported by the Belgian Federal Science Policy office via the PRODEX Program of ESA. A.M. acknowledges funding by the Austrian Science Fund FWF under project numbers P23586 and P23006-N16 and by the Austrian Research Promotion Agency FFG under project number FA 538019. R.D. acknowledges support from the Communauté française de Belgique - Actions de Recherche Concertées and benefits from a European Southern Observatory studentship. We made use of the NASA/IPAC Infrared Science Archive, which is operated by the Jet Propulsion Laboratory, California Institute of Technology, under contract with the National Aeronautics and Space Administration and of the SIMBAD database, operated at CDS, Strasbourg, France.

\section{References}

Baranov, V. B., Krasnobaev, K. V., \& Kulikovskii, A. G. 1971, Sov. Phys. Doklady, 15, 791

Berdyugin, A., \& Piirola, V. 2002, A\&A, 394, 181

Bjorkman, K. S., Miroshnichenko, A. S., McDavid, D., \& Pogrosheva, T. M. 2002, ApJ, 573, 812

Brancewicz, H. K., \& Dworak, T. Z. 1980, Acta Astron., 30, 501

Budding, E., Erdem, A., Çiçek, C., et al. 2004, A\&A, 417, 263

Chaubey, U. S. 1979, Ap\&SS, 64, 177

Coşkunoğlu, B., Ak, S., Bilir, S., et al. 2011, MNRAS, 412, 1237

Collins, II, G. W. 1987, in Physics of Be Stars, eds. A. Slettebak, \& T. P. Snow, IAU Colloq., 92, 3

Comeron, F., \& Kaper, L. 1998, A\&A, 338, 273

Cox, N. L. J., Kerschbaum, F., van Marle, A.-J., et al. 2012, A\&A, 537, A35

Crawford, J. A. 1955, ApJ, 121, 71

de Bruijne, J. H. J., \& Eilers, A.-C. 2012, A\&A, 546, A61

Dehnen, W., \& Binney, J. J. 1998, MNRAS, 298, 387

Deschamps, R., Siess, L., Davis, P. J., \& Jorissen, A. 2013, A\&A, 557, A40

Deschamps, R., Braun, K., Jorissen, A., et al. 2015, A\&A, 577, A55

Draine, B. T. 1981, ApJ, 245, 880

Draine, B. T. 1985 , ApJS, 57, 587

Dunstall, P. R., Fraser, M., Clark, J. S., et al. 2012, A\&A, 542, A50

Gies, D. R., Bagnuolo, Jr., W. G., Ferrara, E. C., et al. 1998, ApJ, 493, 440

Hanuschik, R. W., Hummel, W., Sutorius, E., Dietle, O., \& Thimm, G. 1996, A\&AS, 116, 309

Harmanec, P. 2001, Publications of the Astronomical Institute of the Czechoslovak Academy of Sciences, 89, 9

Hummel, W., \& Štefl, S. 2001, A\&A, 368, 471

Johnson, D. R. H., \& Soderblom, D. R. 1987, AJ, 93, 864

Jorissen, A., Mayer, A., van Eck, S., et al. 2011, A\&A, 532, A135

Kobulnicky, H. A., Lundquist, M. J., Bhattacharjee, A., \& Kerton, C. R. 2012, AJ, 143, 71

Lamers, H. J. G. L. M., Zickgraf, F.-J., de Winter, D., Houziaux, L., \& Zorec, J. 1998, A\&A, 340, 117

Linnell, A. P., Peters, G. J., \& Polidan, R. S. 1988, ApJ, 327, 265

Maercker, M., Mohamed, S., Vlemmings, W. H. T., et al. 2012, Nature, 490, 232

Mastrodemos, N., \& Morris, M. 1999, ApJ, 523, 357

Mayer, A., Jorissen, A., Kerschbaum, F., et al. 2011, A\&A, 531, L4

Mihalas, D., \& Binney, J. 1981, Science, 214, 823

Noriega-Crespo, A., van Buren, D., \& Dgani, R. 1997, AJ, 113, 780

Peri, C. S., Benaglia, P., Brookes, D. P., Stevens, I. R., \& Isequilla, N. L. 2012, A\&A, 538, A108

Peri, C. S., Benaglia, P., \& Isequilla, N. L. 2015, A\&A, 578, A45

Porter, J. M., \& Rivinius, T. 2003, PASP, 115, 1153

Sarna, M. J. 1993, MNRAS, 262, 534

Snow, Jr., T. P. 1981, ApJ, 251, 139

Ueta, T., Speck, A. K., Stencel, R. E., et al. 2006, ApJ, 648, L39

van Buren, D., \& McCray, R. 1988, ApJ, 329, L93

van Leeuwen, F. 2007, A\&A, 474, 653

van Rensbergen, W., de Greve, J. P., Mennekens, N., Jansen, K., \& de Loore, C. 2011, A\&A, 528, A16

Weaver, R., McCray, R., Castor, J., Shapiro, P., \& Moore, R. 1977, ApJ, 218, 377

Wilkin, F. P. 1996, ApJ, 459, L31

Wright, E. L., Eisenhardt, P. R. M., Mainzer, A. K., et al. 2010, AJ, 140, 1868

Zharikov, S. V., Miroshnichenko, A. S., Pollmann, E., et al. 2013, A\&A, 560, A30 


\section{Appendix A: List of objects}

Table A.1. Seventy Algols and Algol-like Be stars with a WISE source counterpart sorted by declination.

\begin{tabular}{|c|c|c|c|c|}
\hline $\mathrm{V}^{*}$ name & 2MASS & $\mathrm{HD} / \mathrm{BD}$ & Spec. typ & Ref. \\
\hline BP Mus & J12503772-7146186 & & & \\
\hline DW Aps & $233003-675$ & HD 156 & B6III & 2,3 \\
\hline EP TrA & $492013-0413$ & HD & A0 & 2,3 \\
\hline Tuc & J23302225-5825346 & HD 22 & A5III & 2,3 \\
\hline R Ara & & HD 149730 & B9IV 1 & $, 2,3$ \\
\hline UZ Nor + & $\mathrm{J} 16281156-5319215$ & & $\mathrm{~B}$ ? & \\
\hline & $7-531$ & HD 100987 & B8IV & 2,3 \\
\hline & 970-5208458 & HD 32011 & $\mathrm{~A} 1 \mathrm{~V}$ & 2,3 \\
\hline T & 1446-5042405 & & B3 & $\mathrm{J}$ \\
\hline KV Pup & -483 & HD 63562 & A0IV & 2,3 \\
\hline$T_{T} \boldsymbol{T}$ & 56 & & & \\
\hline & & HD 80692 & A0III & 2,3 \\
\hline & & HD 1 & A4III & 2,3 \\
\hline & & & 2.5 & ? \\
\hline & & HD & F3IV & 2,3 \\
\hline & $\mathrm{J}$ & & $\mathrm{A} 2 \mathrm{~V}$ & \\
\hline A & J & & A2 & 2,3 \\
\hline $\mathrm{p}+$ & 162 & HD 41511 & A1V & \\
\hline & -1533009 & HD 166126 & F5III 1 & $, 2,3$ \\
\hline$t+$ & J18253147-1241241 & HD 169515 & O9.7Ibep & 2,3 \\
\hline & & $\mathrm{HD}$ & A3 & 2,3 \\
\hline & 3-1049281 & HD & Ape & 1 \\
\hline & & BD- & A2 & 2,3 \\
\hline 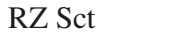 & & $\mathrm{HD}$ & B3Ib 1 & $1,2,3$ \\
\hline & 34 & $\mathrm{H}$ & A2 & 2,3 \\
\hline Ion *+ & & & 2 Vne & 1 \\
\hline & & & K0II & 2,3 \\
\hline & & HD & B4IV 1 & $, 2,3$ \\
\hline & & & G4IV & \\
\hline & $\mathrm{J} 22$ & HD 2 & $\mathrm{~B} 1 \mathrm{Ve}$ & \\
\hline & $3+0141311$ & & A8 & 2,3 \\
\hline & & & A2 & 2,3 \\
\hline & & & B2III & 1 \\
\hline & & & A2e 1 & $, 2,3$ \\
\hline & & HD & A5 & \\
\hline & & & & \\
\hline & & & 30 & 2,3 \\
\hline & & & F5 & 2,3 \\
\hline & & 32 & $7.5 \mathrm{~V} 1$ & $, 2,3$ \\
\hline & & & F6V & 2,3 \\
\hline & & & $35 \mathrm{~V}$ & 2,3 \\
\hline & & 7 & A5 & 2,3 \\
\hline & & & $38 \mathrm{Ve} 1$ & $1,2,3$ \\
\hline & & HD 136175 & B6V 1 & $1,2,3$ \\
\hline & & & & \\
\hline & & $\mathrm{HD}$ & $\mathrm{A} 0$ & $, 2,3$ \\
\hline & & & $9.5 \mathrm{~V}$ & 2,3 \\
\hline $\mathrm{V}$ & & $\mathrm{H}$ & 3Ibep & 1,2 \\
\hline & & $\mathrm{H}$ & A5 & 2,3 \\
\hline * * & 209553 & $H D$ & B1Vne & $, 2,3$ \\
\hline & & $\mathrm{HD} 2$ & ? & $, 2,3$ \\
\hline & 36 & HD 93033 & B8V 1 & $, 2,3$ \\
\hline & 8 & & & 3 \\
\hline & 24 & & B8 & \\
\hline $\mathrm{S}$ & 81 & 1ID & A2 & $, 2,3$ \\
\hline & & HD & B9 & 2,3 \\
\hline RY Per & J02454210+4808379 & HD 17034 & B8V 1 & 2,3 \\
\hline
\end{tabular}

Notes. Objects marked with an asterisk $(*)$ show extended emission in WISE W4, while the plus sign (+) marks objects with a WISE W4/W1 flux ratio which is above the black-body law (see Table 6 in D15).

References. References: (1): Harmanec (2001); (2): Brancewicz \& Dworak (1980); (3): Budding et al. (2004).
Table A.1. continued.

\begin{tabular}{lrrrr}
\hline \hline V* name & 2MASS & HD/BD Spec. type & Ref. \\
\hline KX And & J23070621+5011324 HD 218393 & B3pe & 1 \\
$\varphi$ Per *+ & J01433964+5041192 & HD 10516 & B1.5Ve & 1 \\
AY Per & J03102513+5055543 HD 232756 & B9 & 2,3 \\
CX Dra *+ J18464309+5259166 HD 174237 & B2.5Ve & 1,3 \\
V442 Cas + J23401479+5357339 & A0 & 3 \\
SX Cas & J00104207+5453293 HD 232121 & B5 & $1,2,3$ \\
DM Per & J02255800+5606099 HD 14871 & B5V & 2,3 \\
GG Cas & \multicolumn{1}{c}{ BD +55 274 } & B5 & 2,3 \\
RX Cas & J03074573+6734387 & A5III & 2,3 \\
XY Cep & J23523291+6856015 & B8 & 2,3 \\
SS Cam & J07162474+7319570 & G1III & 2,3 \\
XZ Cam & J05171266+7550053 & A0 & 2,3 \\
RS Cep & J05060320+8014524 & A5III 1, 2, 3 \\
TY UMi & J15175751+8351340 HD 138818 & F0 & 3 \\
\hline
\end{tabular}

